\title{
Impact of Comet Shoemaker-Levy 9 on Jupiter
}

\author{
Thomas J. Ahrens, Toshiko Takata, John D. O'Keefe \\ Lindhurst Laboratory of Experimental Geophysics, Seismological Laboratory 252-21, California Institute of Technology, \\ Pasadena, CA 91125
}

Glenn S. Orton

Jet Propulsion Laboratory, Pasadena, CA 91109

\begin{abstract}
Three-dimensional numerical simulations of the impact of Comet Shoemaker - Levy 9 on Jupiter and the resulting vapor plume expansion were conducted using the Smoothed Particle Hydrodynamics (SPH) method. An icy body with a diameter of $2 \mathrm{~km}$ can penetrate to an altitude of $-350 \mathrm{~km}(0 \mathrm{~km}=1 \mathrm{bar})$ and most of the incident kinetic energy is transferred to the atmosphere between $-100 \mathrm{~km}$ to $-250 \mathrm{~km}$. This energy is converted to potential energy of the resulting gas plume. The unconfined plume expands vertically and has a peak radiative power approximately equal to the total radiation from Jupiter's disc. The plume rises a few tens of atmospheric scale heights in $\sim 10^{2}$ seconds. The rising plume reaches the altitude of $-3000 \mathrm{~km}$, but no atmospheric gas is accelerated to the escape velocity ( $\sim 60$ $\mathrm{km} / \mathrm{s})$.
\end{abstract}

\section{Introduction}

Fragments of Comet Shoemaker-Levy-9 (SL9) are predicted to impact Jupiter in July 1994 [Shoemaker et al., 1993; Yeomans and Chodos, 1993]. Observations using the Hubble Space Telescope indicate the maximum and minimum fragment diameters of $\sim 4$ and $2 \mathrm{~km}$ for the 11 largest fragments [Weaver et al., 1994]. Best estimates of total energy released to the Jovian atmosphere upon impact of all of the comet fragments (CF's) is $10^{29}$ to $10^{31} \mathrm{erg}$, however, the actual mass of CF's are very uncertain.

\section{Entry and Hydrodynamic Instabilities}

We modeled the impact of CF's onto Jupiter in threedimensions in a constant Jupiter gravity field $\left(2325 \mathrm{~cm} / \mathrm{s}^{2}\right)$ using the Lagrangian method called Smoothed Particle Hydrodynamics (SPH) [Gingold and Monaghan, 1977]. A Tillotson equation of state for ice (density, $1.0 \mathrm{~g} / \mathrm{cm}^{3}$ ) was used for the cometary material [O'Keefe and Ahrens, 1982]. We assume the atmospheric structure inferred from Voyager data [Lindal et al., 1981] and extend our model adiabatically into the interior of Jupiter (using 1.4 for the diatomic

1 Lindhurst Laboratory of Experimental Geophysics, Seismological Laboratory 252-21, California Institute of Technology, Pasadena, California

2 Jet Propulsion Laboratory, Pasadena, Califomia

Copyright 1994 by the American Geophysical Union.

Paper number 94GL01325

0094-8534/94/94GL-01325\$03.00 molecule ratio of specific heats). We performed calculations for two sizes of CF's, 2 and $10 \mathrm{~km}$ in diameter with an impact velocity of $60 \mathrm{~km} / \mathrm{s}$ and impact angle of $40^{\circ}$ from the zenith. These sizes characterize the range of maximum sizes and energy of the SL9 fragments as perceived since discovery [Chapman, 1993a; Chapman, 1993b; Scotti and Melosh, 1993; Sekanina et al., 1993; Weaver et al., 1994].

Jovian atmospheric space is segmented into successive overlapping target boxes. In the calculation we assumed 280 comet particles, initially in a sphere, and allowed them impact and then flow through a series of 17 and 36 target boxes, for the 10- and 2-km cases, respectively. Each box contains $\sim 50,000$ atmospheric "particles". The calculation was terminated when all the comet "particles" came to rest at various points along the trajectory. The dimensions of the cuboid boxes are 80 and $20 \mathrm{~km}$ for the $10-$ and $2-\mathrm{km} \mathrm{CF's,}$ respectively, and the mass of particles were increased with depth to take into account atmospheric structure. A number of studies concerning the present numerical methods, including the effect of varying the number of SPH particles used to represent the cometary material are still in progress. Detailed description of our calculations is given in Takata et al. [1994].

When the bolide enters Jupiter's atmosphere it induces a high temperature shock wave in the gas in front of the bolide. The overall mechanical properties of comet nucleii are unknown, but much evidence indicates they are weak. We assume comet nucleus rheology can be approximated by a viscous fluid. We assume the lower limit to viscosity is that of ice-grain sliding, and thus the viscosity is similar to liquid water. The radiation and convective flow of the shocked atmosphere around the front melts and ablates small quantities of material from the bolide. The high speed flow of the atmosphere tangential to the melted material drives surface Kelvin-Helmholtz (K-H) instabilities that grow and shed melt droplets into the flow behind the body. These particles are micron-sized, as the fastest growing wavelengths of the $\mathrm{K}-\mathrm{H}$ instabilities are microns in length. The relative mass loss for large impactors via ablation processes (e.g. surface melting and shedding of particles and vaporization) is small [Bronshten, 1983]. We believe the destruction of cometary fragments at higher altitudes found by Sekanina [1993] results from his assuming an ablation coefficient which is too large. However, wavelengths much shorter -1 micron are suppressed by the viscosity of the melt and do not grow --this is seen in the turnover in the curves in Figure 1 calculated using the formulation of Keith and Banks [1990].

As the bolide penetrates further into the atmosphere, dynamic pressures within the shocked material in front of the 


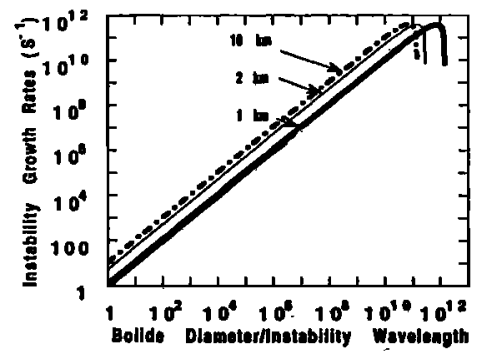

Fig 1 Growth rate of Kelvin-Helmboltz instabilities as a function of the diameter of the impactor divided by the instability wave length. The curves are for impactor diameters of 1,2 , and $10 \mathrm{~km}$ and the depth into the atmosphere is $300 \mathrm{~km}$ relative to the 1 bar level of Jupiter.

bolide exceed the strength of the impactor. This results in fracturing and then subsequent radial deformation of the bolide. The aerodynamic stresses acting on the bolide are greatest at the center of the front surface fragment and give rise to the spreading and flattening of the bolide [MacLow and Zahnle, 1994; Melosh, 1989; Vickery, 1993; Zahnle, 1992]. The ratio of the deformed CF cross-sectional area to the initial sphere was found in SPH calculations to increase from unity to a maximum of -2.3 for a $1 \mathrm{~km}$ radius impactor (see Figure 2b) and -1.7 cross-sectional area for a $5 \mathrm{~km}$ radius impactor. Qualitatively, this type of distortion and flattening has been observed in laboratory experiments of breakup of liquid droplets by gas streams [Ranger and Nichols, 1970]. We infer that such hydrodynamic instabilities and the surrounding bow shock prevent the projectile from expanding to the effective diameters that are as large as -20 times the undisturbed diameter predicted by models based only on inertial confinement [Zahnle, 1992].

Besides $\mathrm{K}-\mathrm{H}$ instabilities, Rayleigh-Taylor (R-T) instabilities occur and these are driven by the deceleration forces acting on the bolide. The particles ejected ahead of the main bolide (Figure 2a) are believed to result from R-T instabilities in our calculations. In general, we find from analytic modeling the time for $R-T$ instabilities to grow relative to the characteristic deceleration time, $t_{d e c e l}$, is given by

$$
\mathrm{t}_{\mathrm{R}-\mathrm{T}} / \mathrm{t}_{\text {decel }}=0.23(\lambda / \ell)^{1 / 2}
$$

where $\lambda$ is the instability wavelength, and $\ell$ is atmospheric scale height. Ivanov et al. [1992] observed that upon passage of an impactor through the Venusian atmosphere that surface instabilities grow at the front of the projectile and limited the distortion of the projectile. Recently, Crawford et al. [1994] observed similar phenomena in their calculation of SL9 impact on Jupiter.

Moreover, instabilities are driven by velocity differences between the atmosphere and the bolide surface and are most prevalent near the edge of the front face of the bolide. The time for K-H instabilities to grow relative to the bolide characteristic deceleration time is given by

$$
\begin{aligned}
& t_{K-H^{\prime}} / t_{\text {decel }}=0.023 C_{D}-1 / 3\left[\left(\rho_{p} \eta_{p}\right) /\left(\rho_{a} \eta_{a}\right)\right]^{1 / 3} \\
& (R / \ell)^{1 / 3}(\lambda / \ell)
\end{aligned}
$$

where $C_{D}$ the drag coefficient, and $\rho_{a}$ and $\rho_{p}$ are the densities of the atmosphere and bolide, respectively, $\eta_{a}$ and $\eta_{p}$ are the effective viscosities of hydrogen at depths of $\sim 300 \mathrm{~km}$ in
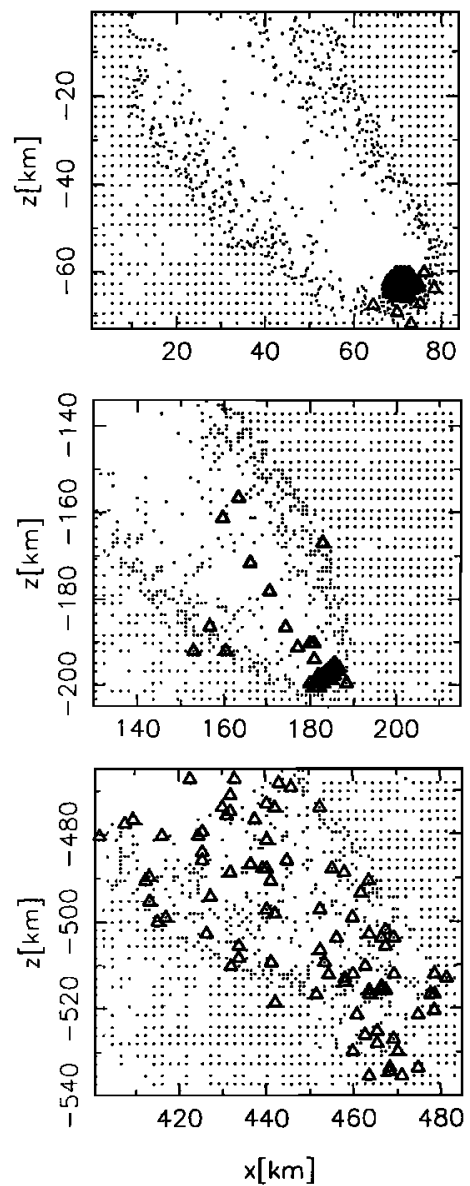

Fig. 2. Smoothed-Particle-Hydrodynamics (SPH) calculations of the penetration of a fragment of Comet Shoemaker-Levy 9 fragment into Jupiter's atmosphere. The diameter of the bolide is $10 \mathrm{~km}$ and the angle of entry is $40^{\circ}$ from the zenith. Shown are the density fields at various times relative to the time the bolide penetrated to the 1 bar altitude. Dots indicate planetary atmosphere and triangles bolide SPH particles. The times are $2.0,5.0$, and 21 sec., respectively (after Ahrens et al. [1994] ).

Jupiter and the bolide (taken to be that of liquid water).

The final breakup of the bolide occurs when the instabilities having wavelengths on the order of the size of the bolide $(\lambda / R \sim 1)$ grow to large amplitudes. These considerations, generally agree with the computational results of Figure 2. The growth rates for large scale K-H instabilities are on the order of $1 \mathrm{~s}^{-1}$ for bolides $1 \mathrm{~km}$ in radius and on the order of $10^{1} \mathrm{~s}^{-1}$ for bolides 1 to $5 \mathrm{~km}$ in radius. The growth rates for $\mathbf{R}-\mathrm{T}$ instabilities are similar. The present SPH calculations show disintegration occurring at time scales predicted from these growth rates. Shown in Figure $2 c$ is final disintegration and the stopping of the disintegrated fragments.

Penetration is complete when CF SPH particles stop. CF's can penetrate to an altitude of $-350 \mathrm{~km}(-200$ bar total atmospheric pressure), for the initial diameter for the $D=2$ $\mathrm{km}$ case and to the altitude of $-550 \mathrm{~km}(-800 \mathrm{bar})$, for the $D$ $=10 \mathrm{~km}$ case. The energy transfer from the CF to the atmosphere occurs mostly in the altitude range from $-100 \mathrm{~km}$ $(-10 \mathrm{bar})$ to $-250 \mathrm{~km}(-100 \mathrm{bar})$ in the case of $\mathrm{D}=2 \mathrm{~km}$ and $-350 \mathrm{~km}(-200 \mathrm{bar})$ to $-480 \mathrm{~km}(-500 \mathrm{bar})$ in the case of $D$ $=10 \mathrm{~km}$ (Fig. 3). 


\section{The Atmospheric Plume}

We subsequently carried out 3-dimensional calculations (with Jupiter's gravity) of the plume expansion (Fig. 4). We distributed $-10^{5}$ atmospheric particles in model atmospheric box with a 500 by $500 \mathrm{~km}$ footprint extending from 350 to $-400 \mathrm{~km}$ altitude in the case of the $2-\mathrm{km}$ diameter CF. The energy density of deposition shown in figure 3 is placed on $\sim 20$ atmospheric particles along the trajectory. In $\sim 10^{2}$ seconds after the impact, the resulting plume rises a few tens of atmospheric scale heights and it expands vertically rather than horizontally in an inhomogeneous atmosphere. Both the density gradient in the atmosphere and the expansion of compressed gas result in the upward motion of the plume. Some $20 \%$ of the energy deposited in the deep atmosphere can be transported above $-100 \mathrm{~km}$ in 100 seconds and most of the energy is brought up above $100 \mathrm{~km}$ in -200 seconds. A mass of atmospheric gas equal to -10 times the CF mass is transported from below the cloud deck to above $\sim 100 \mathrm{~km}$ in $10^{2}$ seconds and a total of $\sim 20$ times the initial CF mass is elevated above $100 \mathrm{~km}$. The rising plume achieves an altitude of $\sim 3000 \mathrm{~km}$, however, no atmospheric gas (in our calculation) is accelerated to escape velocity. The plume transport results in the vertical mixing of deep atmospheric constituents, such as $\mathrm{NH}_{3}, \mathrm{H}_{2} \mathrm{O}$, and possibly $\mathrm{H}_{2} \mathrm{~S}$, in addition to the vaporized cometary materials. Subsequent condensation of both deep atmospheric and cometary materials may be a source of particulates in the upper atmosphere.

\section{Radiative Signatures}

The radiation upon entry from the heated atmosphere and vaporized cometary media within the temporary conical cavity will be multiply Rayleigh scattered by the $\mathrm{H}_{2}$ and $\mathrm{He}$ of Jupiter's atmosphere, and obscured by the several Jovian cloud decks. Moreover, the radiant flux may also be reflected from one or more Galilean satellites depending upon their position during impact. The observed temperature during entry of a cometary fragment into Jupiter's atmosphere will be in excess of $10^{4} \mathrm{~K}$. The details of the spectrum will depend on the degree of ionization which occurs in the bolide material and in Jupiter's atmosphere [Chevalier and Sarazin, 1994; MacLow and Zahnle, 1994]. In any case, the $\sim 10^{4} \mathrm{~K}$ temperature seen in the impact flash during bolide

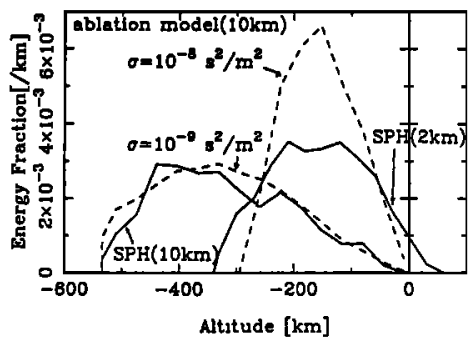

Fig. 3. The fractional energy deposition of the initial kinetic energy of the comet as a function altitude in the case of 2 and $10 \mathrm{~km}$ diameter-cometary fragments. The results of SPH calculation are shown by the solid lines, and the results of meteoric ablation model with the ablation coefficients, $\sigma$, of $10^{-9}$ and $10^{-8} \mathrm{~s}^{2} / \mathrm{m}^{2}$ [Bronshten, 1983] for $\mathrm{D}=10 \mathrm{~km}$, are shown by dashed lines. These values compare to $2 \times 10^{-7}$ $\mathrm{s}^{2} / \mathrm{m}^{2}$ assumed by Sekanina [1993]. Note that $\sigma \sim 10^{-9} \mathrm{~s}^{2} / \mathrm{m}^{2}$ is the best fit to the SPH result.

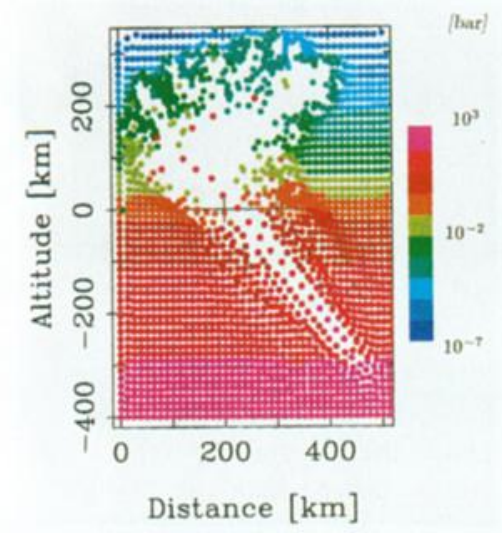

Fig. 4. The pressure of atmospheric particles upon plume expansion of $D=2 \mathrm{~km}$ cometary fragment after $75.2 \mathrm{sec}$ (after Ahrens et al. [1994]).

entry will occur (because of radiative cooling) for only a minute or so.

Because the optical radiation from the $(\sim 10 \mathrm{sec})$ entry of a SL9 fragment into Jupiter will emit radiation anisotropically, we chose to model only the radiation from the second SPH calculation describing the plume. Moreover, the entry flash may be partially obscured in many directions by the asymmetry of the entry hole, opacity of ionized gas, and the $\mathrm{NH}_{3}, \mathrm{NH}_{4} \mathrm{SH}$, and $\mathrm{H}_{2} \mathrm{O}$ cloud layers.

Starting with plume calculations of 2 -and $10-\mathrm{km}$ SL9 fragments, we have considered each particle as a greybody radiator disc (with a specific heat of $5 R / 3, R$ is the gas constant) and calculated the total normal radiative power as a function of wavelength at a series of times (e.g. Figure 5). Since we are not accounting for ionization, reaction between species, absorption by $\mathrm{CH}_{4}$ or Rayleigh scattering from $\mathrm{H}_{2}$, the present calculations are an upperbound to the actual radiating power. However, most of the hot materials, shown in Figure 4 are at, or above, the elevations such that there will be minimal absorption effects of clouds. We observe that in the case of the $2-\mathrm{km}$ comet nucleus fragment impact that its particle velocity is such that it should achieve an altitude of $3000 \mathrm{~km}$ within several minutes.

\section{Predictions}

In July 1994, the Galileo spacecraft will be in a good position (1.6 AU away from Jupiter) to observe the plumes from SL9 impact collisions with Jupiter with the Solid State Imaging Experiment [Belton et al., 1992], the Near-Infrared Mapping Spectrometer experiment [Carlson et al., 1992], the Photo-polarimeter Radiometer experiment [Russell et al., 1992], and the Ultraviolet Spectrometer experiment [Hord et al., 1992]. The flux emitted by the plume associated with a $2-\mathrm{km}$ diameter fragment is some $30 \%$ of the solar visible flux reflected by Jupiter. It is comparable to both Jupiter's reflected solar flux near 1 micron and an order of magnitude or more higher than Jupiter's near-infrared reflected flux, and Jupiter's average 5- and 7.8-micron thermal emission. Using our 2 -and $10-\mathrm{km}$ results, we infer that the plume from impact of a $2-\mathrm{km}$ diameter fragment yields a radiant power equivalent to $3 \%$ of Jupiter's total flux and some $10 \%$ of Jupiter's average thermal emission at 5 and 7.8 microns. Thus, observations by Galileo instruments are technically feasible for a large number of the fragments if they are as dense as we have assumed. 


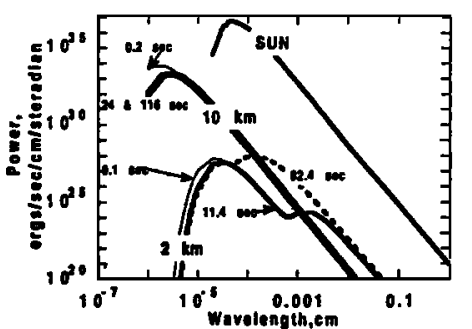

Fig. 5 Calculated spectra from Shoemaker-Levy 9 impactinduced plumes for 2 and $10 \mathrm{~km}$ diameter fragments. The peak power from the $2 \mathrm{~km}$ fragments radiates at the approximately same power level as the total Jupiter disk. The total power from the sun, considered as a point source is shown, for comparison.

Direct earth-based observations of the impact-induced plume may be possible as it will rise above Jupiter's SW horizon as seen from the earth (prior to dawn at the impact point) on a time scale of 10 to 100 secs. Moreover, observation of the inner Galilean satellites brightnesses as a function of time may detect a reflection of the plume illumination superimposed on the solar illumination. The plume generated by impact of a $2-\mathbf{k m}$ diameter fragment would increase Io's visible flux by some $10 \%$ in the visible and by $1 \%$ or less in the near infrared. The plume generated by the $0.5-\mathrm{km}$ fragment illuminates Io (for normal incidence) only $0.01-0.03 \%$ more than the sun between the visible and near infrared. However, at wavelengths of 5 microns and longer, the $0.5 \mathrm{~km}$ fragment induced plume illuminates Io some $1-3 \%$ more than the sun. Clearly, the most favorable spectral band for indirect detection of impart-induced plumes is in the near or middle infrared.

For SL9 fragments with diameters $<1 \mathrm{~km}$, impact-induced plume brightnesses are expected to be decreased by plume and atmospheric opacity effects [Chevalier and Sarazin, 1994]. However, upon impact of smaller objects in Jupiter's atmosphere, the region of maximum energy deposition has been demonstrated to occur at higher elevations [Takata et al., 1994]. As a result, the radiated power levels in the resulting plume decrease with impactor energy, E, (ergs) at a lower rate than might be expected. Recent studies of the plume radiant power, $P$ (ergs/sec/cm/steradian) at $400 \mathrm{~nm}$ from a $400 \mathrm{~m}$ diameter comet fragment when taken with calculations shown in Fig. 5 , can be fit with $\log _{10} P=7.41$ $+0.715 \log _{10} E$ with a deviation parameter, $R=0.93$.

\section{Acknowledgments.}

D. Stevenson, J. Spencer, K. Zahnle, T. Johnson, and G. Ravichandran provided many helpful suggestions which we appreciate. We thank K. Zahnle and M. MacLow, and G. Field and A. Ferrara for their preprints, and P. Weissman, $M$. A'Hearn, K. Ziemelis, and two anonymous reviewers for helpful comments. Research supported by NASA and Cray Research Corp. Contribution number 5381, Division of Geological and Planetary Sciences.

\section{References}

Ahrens, T. J., T. Takata, J. D. O'Keefe, and G. S. Orton, Comet Shoemaker-Levy-9 impact on Jupiter, Nature, submitted, 1994.

Belton, M., K. P. Klaason, M. P. Clary, J. L Anderson, and C. O. Anger, The Galileo solid-state imaging experiment, Space Sci. Rev., 60, 413455, 1992.
Bronshten, V. A., Physics of Meteoric Phenomena, D. Reidel Publishing Co., Boston, 1-356 pp., 1983.

Carlson, F., P. R. Weissman, W. D. Smythe, J. C. Mahoney, and I. Aptaker, Near-infrared mapping spectrometer experiment on Galileo, Space Sci. Rev., 60, 457-502, 1992.

Chapman, C. R., Comet on target for Jupiter, Nature, 363, 492-493, 1993a.

Chapman, C. R., Preparing for the comet crash, Nature, 365, 784-785, $1993 b$.

Chevalier, R. A., and C. L. Sarazin, Explosions of infalling comets in Jupiter's atmosphere, Astrophys. J., in press, 1994.

Crawford, D. A., T. G. Trucano, M. B. Boslough, M. E. Kipp, and J. M. McGlaun, The influence of body shape on the deformation and break-up of Comet Shoemaker-Levy 9 fragments as they enter the Jovian atmosphere, Lunar \& Planet. Sci. (abstract), XXV, 295-296, 1994.

Gingold, R. A., and J. J. Monaghan, Smoothed particle hydrodynamics: theory and application to non-spherical stars, Month. Not. R. Astr. Soc., 181, 375-389, 1977.

Hord, C. W., W. E. McClintock, A. I. F. Stewart, C. A. Barth, and L. W. Esposito, Galileo ultraviolet spectrometer experiment, Space Sci. Rev., 60, 503-530, 1992.

Ivanov, B. A., I. V. Nemchinov, V. A. Svetsov, A. A. Provalov, V. M. Khazins, and R. J. Phillips, Impact cratering on Venus: Physical and mechanical models, J. Geophys. Res., 97, 16,167-16,182, 1992.

Keith, J. C., and N. E. Banks, Hypervelocity impact and aerodynamic breakup of liquids, Int. J. Impact Engng., 10, 309-322, 1990.

Lindal, G. F., G. E. Wood, G. S. Levy, J. D. Anderson, D. N. Sweetnam, H. B. Hotz, B. J. Buckles, D. P. Holmes, P. E. Poms, V. R. Eshelman, G. L. Tyler, and T. A. Croft, The atmosphere of Jupiter: An analysis of the Voyager radio occultation measurements, J. Geophys. Res., 86, 8721-8727, 1981.

MacLow, M.-M., and K. Zahnle, The collision of Jupiter and Comet Shoemaker-Levy-9, Icarus, in press, 1994.

Melosh, H. J., Impact Cratering, A Geologic Process, Oxford University Press, New York, 1-245, 1989.

O'Keefe, J. D., and T. J. Ahrens, Cometary and meteorite swarm impact on planetary surfaces, J. Geophys. Res., 87, 6668-6680, 1982.

Ranger, A. A., and J. A. Nichols, Shape and surrounding flow field of a drop in a high-speed gas stream, ALAA Joumal, 8, 1720-1722, 1970.

Russell, E. E., F. G. Brown, and R. A. Chandos, Galileo photopolarimeter radiometer, Space Sci. Rev., 60, 531-563, 1992.

Scotti, J. V., and H. J. Melosh, Estimate of the size of Comet ShoemakerLevy 9 from a tidal breakup model, Nature, 365, 733-735, 1993.

Sekanina, Z., Disintegration phenomena expected during collision of Comet Shoemaker-Levy 9 with Jupiter, Science, 262, 382-387, 1993.

Sekanina, Z., P. W. Chodos, and D. K. Yeomans, Tidal disruption and the appearance of periodic Comet-Shoemaker-Levy-9, Astron. J., in press, 1993.

Shoemaker, C. S., E. M. Shoemaker, and D. H. Levy, Comet ShoemakerLevy, IAU Circ., 5725, 1993.

Takata, T., J. D. O'Keefe, T. J. Ahrens, and G. S. Orton, Comet Shoemaker-Levy-9: Impact on Jupiter and plume evolution, Icarus, in press, 1994.

Vickery, A., Numerical simulation of a comet impact on Jupiter, EOS, 74, 391, 1993.

Weaver, H. A., P. D. Feldman, M. F. Ahearn, C. Arpigny, and R. A. Brown, Hubble-Space Telescope observations of CometP/Shoemaker-Levy 9 (1993e), Science, 263, 787-791, 1994.

Yeomans, D. K., and P. Chodos, Minor Planet Circ. No., 22197, 1993.

Zahnle, K., Airburst origin of dark shadows on Venus, J. Geophys. Res., 97, 10243-10255, 1992.

T. J. Ahrens, T. Takata, and J. D. OKeefe, Lindhurst Laboratory of Experimental Geophysics, Seismological Laboratory 252-21, California Institute of Technology, Pasadena, CA 91125

G. S. Orton, Jet Propulsion Laboratory, Pasadena, CA 91109

(Received February 4, 1994; revised May 4, 1994; accepted May 5 , 1994.) 